\title{
Junior doctor-designed induction booklet to improve future junior doctor experience in a new post
}

\author{
Authors: Nibu Thomas, Eoin McGrann, Louise Zammit, Patrick Eaton, William White, Claire Lingam, Charlotte \\ Thompson, Christopher Morgan, Linda William, Philip Davie, Rachel Shellien and Ronan O'Neill
}

\section{Introduction}

Starting your rotation as a junior doctor on an unfamiliar ward can be challenging, regardless of your grade. There is evidence showing increased mortality of $4.3-12 \%$ and $0.3-7.2 \%$ longer lengths of stay around changeover dates. ${ }^{1}$ High quality of induction was one of the recommendations by the Academy of Medical Royal Colleges for safe trainee changeover. ${ }^{2}$ Our project by a team of junior doctors was aimed at improving the initial experience of the future junior doctors on the same ward at a university hospital. The tool used was an induction booklet that the doctors could refer to from the first day and re-refer to it as the rotation progressed.

\section{Methods}

PDSA Cycle 1: Informal discussions were held and we conducted a survey of the junior doctors to assess needs. The results from the survey were analysed and a solution suggested was a brief induction booklet by junior doctors. This booklet of 4 pages was designed and written by junior doctors from the ward.

PDSA Cycle 2: The new induction booklet was trialled in December 2018 with the new cohort of junior doctors, who were then surveyed in January 2019.The results were analysed and the booklet was modified further. The new version will be trialled in April 2019.

\section{Results and discussion}

Seven junior doctors took part in the first survey and five in the second. Each cohort was asked nine questions and to score from seven grades, namely a) very easy, b) easy, c) somewhat easy, d) neither easy nor difficult, e) somewhat difficult, f) difficult, and g) very difficult.

In the first survey, $28 \%$ of respondents found it somewhat difficult to understand ward timetable (MDT meetings, consultant ward rounds etc) prior to the introduction of the booklet, but this reduced to $0 \%$ after the intervention, with $80 \%$ rating the ward timetable as easy or very easy to understand.
Prior to the intervention, $14 \%$ of respondents found it difficult and $28 \%$ found it somewhat difficult to understand their role in an acute stroke thrombolysis alert; after introduction of the booklet, this reduced to $20 \%$ who found it somewhat difficult. Pre-intervention, $42 \%$ found it somewhat difficult to request the right investigations and post-intervention, this improved to $100 \%$ finding it somewhat easy to very easy. Before the intervention, $14 \%$ said it was difficult and $28 \%$ said it was somewhat difficult to find frequently used phone and bleep numbers, but after the introduction of the booklet, this improved to $100 \%$ finding it very easy to somewhat easy. Fifty-six per cent found it difficult or somewhat difficult to refer to stroke-related specialist teams (vascular surgery, neurology, nutritional team etc) before the booklet was introduced; afterwards, this figure improved to $60 \%$. One hundred per cent of the new cohort of junior doctors answered in the survey that they found the induction booklet helpful.

\section{Conclusion}

There was a clear and definite improvement in the trainee experience after the introduction of the induction booklet. This proves that junior doctors are in an excellent position to improve the experience of other junior doctors and this was done on this occasion with an induction booklet.

We hope to replicate this success on other wards in the hospital, ensuring that the information is local, relevant and up to date.

\section{References}

1 Young JQ, Ranji SRR, Wachter RM et al. 'July effect': impact of the academic year-end changeover on patient outcomes. A systematic review. Ann Intern Med 2011;155:309-15.

2 Academy of Medical Royal Colleges. Recommendations for safe trainee changeover. London: AoMRC, 2013. www.aomrc.org.uk/ reports-guidance/recommendations-for-safe-trainee-changeover-0613/ [Accessed 25 March 2019].

Authors: Aintree University Hospital NHS Foundation Trust, Liverpool, UK 